\title{
AN ANALYSIS OF APP PRIVACY STATEMENTS
}

\author{
Janet Prichard, Bryant University, prichard@bryant.edu \\ Kevin Mentzer, Bryant University,kmentzer@bryant.edu
}

\begin{abstract}
In 2016, there were over 13 billion app downloads worldwide across the iOS App Store and Google Play. Many of these apps collect user and usage data. Apple iTunes requires such apps to post a privacy policy with the app, which is mostly ignored by users given that is it buried at the bottom of the app page when viewed on a mobile device. In 2004, California established a law with broad requirements for privacy policies. Moreover, in 2014, the Attorney General's office in California made further recommendations on the readability of privacy policies. In this paper, we will examine the characteristics of the privacy statements found for popular business apps in the iTunes store by examining the readability metrics and confirm the readability of privacy policies associated with apps. Our study also provides a privacy policy text corpus for further research.
\end{abstract}

Keywords: privacy policy, security, legal issues, End User License Agreement

\section{INTRODUCTION}

Today's smartphone users are tethered to their devices due to the proliferation of both personal and business related applications available for these devices. They use them to instantly communicate with friends and business associates through instant messenger apps such as Beejive and Kik Messenger. They use them to pay for goods through apps such as Venmo and Apple Pay. They use them to find out where they are going through navigation apps such as Waze and Google Maps. They use them to monitor their waking hours through health and fitness apps such as Nike+ and MyFitnessPal, and their sleeping hours by recording their movements and sounds through apps such as Pillow and SleepCycle. Smartphones, and more specifically, the apps on these smartphones, have completely infiltrated our lives.

When we use smartphone apps to perform many of these activities, they often collect personal data about us. Many of these apps require a certain level of data in order to perform their intended purpose, but there is also public concern that unnecessary data is also being collected (Rainie, 2017). A privacy policy is a statement that discloses how an app or website gathers, uses, discloses, and manages a customer or client's data and is intended to help the potential customer to evaluate whether they wish to use any given app. The Apple App Store Review Guidelines (App Store Review Guidelines, 2017) states that "Protecting user privacy is paramount in the Apple ecosystem" and that developers should use care when handling personal data, "Apps cannot use or transmit someone's personal data without first obtaining their permission and providing access to information about how and where the data will be used."

In addition, government agencies have started to take notice of data collection activities on websites and online services. California was the first state in the country to establish a law with broad requirements for privacy policies (California State Legislature, 2003). It applies to websites and online services that collect personally identifiable information about people living in California. The requirements of the law primary pertain to the collection of personally identifiable information and the rights of consumers with respect to that information. Clauses in the law include the following:

(1) The Identification of the categories of personally identifiable information collected

(2) The categories of third-party persons or entities with whom the operator may share that personally identifiable information

(3) If they have a process for consumers to review the information, provide a description of the process.

(4) How consumers are notified of changes in the privacy policy 


\section{Issues in Information Systems}

Volume 18, Issue 4, pp. 179-188, 2017

Since the law went into effect in 2004, the California Attorney General's Office has published further recommendations with respect to privacy policies. (Kamal D. Harris, Attorney General, California Department of Justice, 2014). In this report, they note that consumers do not understand, and often do not read, privacy policies. In response to the concerns, they established guidelines with respect to privacy statements, which include the following:

- Use plain, straightforward language. Avoid technical or legal jargon.

- Use short sentences. Use the active voice.

- Use a format that makes the policy readable, including on smaller screens, such as on a mobile device.

The aim of these guidelines is to make privacy policies more readable to the average person. Many studies support these guidelines. For example, when shorter sentences (average sentence length of 14 words) are used, readers comprehend more than $90 \%$ of what they read (Vincent, 2014). When sentence lengths reach 43 words, comprehension drops to 10\%. In the Oxford Guide to Plain English Martin Cutts (2004) suggests: "Over the whole document, make the average sentence length 15-20 words." Sentences with more than 25 words force users to work harder to read the material. Many readability formulas use sentence length as one of the input values. Moreover, plain, straightforward language is often described as plain English. Plain English writing is easy to understand and emphasizes clarity, brevity, and avoidance of overly complex vocabulary (Plain English, 2017). The goal is to be clear and avoid needless technical jargon. It is commonly used in relation to official government or business communication.

In 2012, six companies that comprise the majority of the mobile market (Amazon, Apple, Google, Hewlett-Packard, Microsoft, and Research In Motion) have agreed to the principles of the California law (Mobile app Industry Agrees to Privacy Standards in California., 2012). In 2013, an amendment to the law addressed online tracking as consumers move across websites and online services.

Using the California Attorney General's guidelines, we identify measures to test these guidelines and evaluate how well mobile apps are meeting these requirements. We find, in general, that the privacy policies in general fail at meeting the guidelines. We then separate out those privacy statements that specifically address California state law to determine if that subset of policy statements meet the Attorney General's guidelines. Again, we find little evidence that the guidelines are met even with this subset of policy statements.

This work sets out to discover if the establishment of requirements by states such as California have had any impact on the readability of privacy policies.

\section{RESEARCH METHODOLOGY}

Given the potentially greater concerns over privacy in a business setting, for this study we chose to examine the top free apps in the Apple iTunes App Store Business Category. Our goal was to analyze at least 100 unique privacy statements. Many apps do not collect any personal information on the user, and thus may not have an associated privacy statement. We also note that some apps come from the same company and often utilize the same privacy statement. With these considerations, we collected information on the top 180 free apps in the business category to insure that we would get at least the 100 privacy statements for study.

For each app, we downloaded available privacy, developer, and other statements linked to the site, though our interest in this study is with the privacy policies. Out of the 180 apps selected, 53 did not have a privacy policy associated with them. Two of the statements were in a foreign language (Chinese and Arabic). The one in Chinese did not appear to be a privacy statement when translated. Sixteen of the agreements were duplicates, or near duplicates of other agreements because they came from the same company. Lastly, one app that had a bad link that could not be resolved to a privacy statement. This left 110 statements available for analysis.

Once the privacy statements were captured (most often as HTML documents), they were stripped of the HTML coding and saved as plain text files. These text files were then imported into Microsoft Word which allowed us to capture the basic information about each privacy statement (such as number of characters, words, and paragraphs), and readability 


\section{Issues in Information Systems}

Volume 18, Issue 4, pp. 179-188, 2017

metrics. The privacy statements were then run through a program called Readability Studio (Oleander Software, 2017). This software provides additional readability metrics and graphs.

We used the guidelines as set forth by the California AG's Office to evaluate the privacy policies (see Table 1 for a summary of these measures). To evaluate the first guideline (use plain, straightforward language) we utilized the Flesch Reading Ease score and the Flesch-Kincaid Grade Level. To evaluate the second guideline (use short sentences, use the active voice.) we utilized the total number of words, words per sentence, words per paragraph, the Fry Test, and the percentage of passive voice sentences. To measure the final guideline (use a format that makes a policy readable, including on smaller screens, such as on a mobile device) we used a predicted Cloze Score and an estimate of the total number of swipes needed to read the policy. The result of this analysis will provide an overall indication on whether the policy is consumable by the apps users.

Table 1. Guidelines and Measurements

\begin{tabular}{|l|}
\hline Guideline 1: Use plain, straightforward language. Avoid technical or legal jargon. \\
\hline $1.1 \quad$ Flesch Reading Ease \\
\hline 1.2 Flesch-Kincaid Reading Level \\
\hline Guideline 2: Use short sentences. Use the active voice. \\
\hline $2.1 \quad$ Average Number of Words Per Sentence \\
\hline 2.2 Average Number of Sentences Per Paragraph \\
\hline $2.3 \quad$ Fry Test \\
\hline 2.4 Percentage of Passive Voice Sentences \\
\hline $\begin{array}{l}\text { Guideline 3: Use a format that makes the policy readable, including on smaller screens, such as on a mobile } \\
\text { device. }\end{array}$ \\
\hline 3.1 Predicted Cloze Score \\
\hline 3.2 Number of swipes to read entire text \\
\hline
\end{tabular}

\section{RESULTS}

The Spelling \& Grammar feature in Microsoft Word was used to gather basic information about each document. A summary of those results is shown in Table 2. Note that in addition to providing word, sentence, and paragraph counts, Microsoft Word also provides two popular readability metrics, the Flesch Reading Score and Flesh-Kincaid Reading Ease. Both tests use the same core measures (word length and sentence length), but with different weighting factors.

We used two measures to evaluate Guideline 1, which included straightforward language and avoiding technical jargon.

The first measure was the Flesch Reading Ease score, The Flesch Reading Ease measurement scores text on a range from zero (very hard) to 100 (very easy), so the lower the score, the more difficult the text is to understand. A score of 100 means the content is very simple and easily understood. A score of 60-70 indicates the content is suitable for an intermediate reader at a grade level of 8 and above. A grade of 0-30 requires advanced skills with the reader having a university level reading skill. The Flesch Reading Ease Scores for the privacy policies ranged from 16.2 (advanced reading level) to 57.7 (slightly higher than intermediate level) with a mean of 34.8 , indicating that many privacy policies require college level reading skills.

The second measure of readability, the Flesh-Kincaid Grade Level, represents a grade level or number of years of education in the United States needed to comprehend the text. The scores for the privacy policies ranged from 9.6 to 17.3 with a mean of 14.0, again indicating college level reading skills. The maximum Flesh-Kincaid Reading Ease found in the documents had a value of 17.3, meaning that someone would need education beyond a bachelor's degree to reasonably understand the document. 


\section{Issues in Information Systems}

Volume 18, Issue 4, pp. 179-188, 2017

We used four measures to evaluate Guideline 2, which included the recommendation of shorter sentences and the use of the active voice.

First was the number of words per sentence. The number of words per sentence ranged between 14.4 and 31.5 with an average of 24 . The number of words per privacy policy ranged from 186 to 30,554 with an average of 3133 . Second was the number of sentences per paragraph ranged from 1.2 to 5.5 with an average 2.7 sentences. Third, was the Fry Test, which is similar to the Flesch-Kincaid score, in that it produces a grade level of education needed to comprehend the text. The Fry Test scores for the privacy policies ranged from 10 to 17 with an average of 15.8 indicating that several years of university education was required in order to understand the text.

Fourth, we examined the percentage of sentences written in active versus passive voice. Active voice helps readers keep clear the logic of who did what to whom. Passive voice should be avoided due to the impersonal tone it presents, and causing the reader to focus on the wrong component of the sentence (Felker, 1981). The percentage of passive voice sentences $2 \%$ to $39 \%$ with an average of $15 \%$.

We used two measures to evaluate Guideline 3, policy readability. First was the Cloze score (Taylor, 1953). This metric has been used to assess the comprehension of privacy statements on mobile devices (Singh, 2011). Mobile devices present unique challenges in reading privacy policies due to the small screen size, lack of a standardized interface, and environments that are not conducive to reading. The Cloze test presents the reader text with some of the words left out, and the reader replaces the missing words. The findings by Singh, et.al. concluded that no readers were able to comprehend privacy policies when presented on a mobile device. They also found a correlation between the length of the document and the readers Cloze score, longer documents were more difficult to comprehend. The study also noted that in general, Cloze scores indicated that privacy policies were more readable on desktop environments than on mobile devices. Hence, mobile environments have an adverse impact on the readability of privacy policies (see Figure 1). The Cloze score for the privacy statements ranged from 23 to 36 with an average of 28.

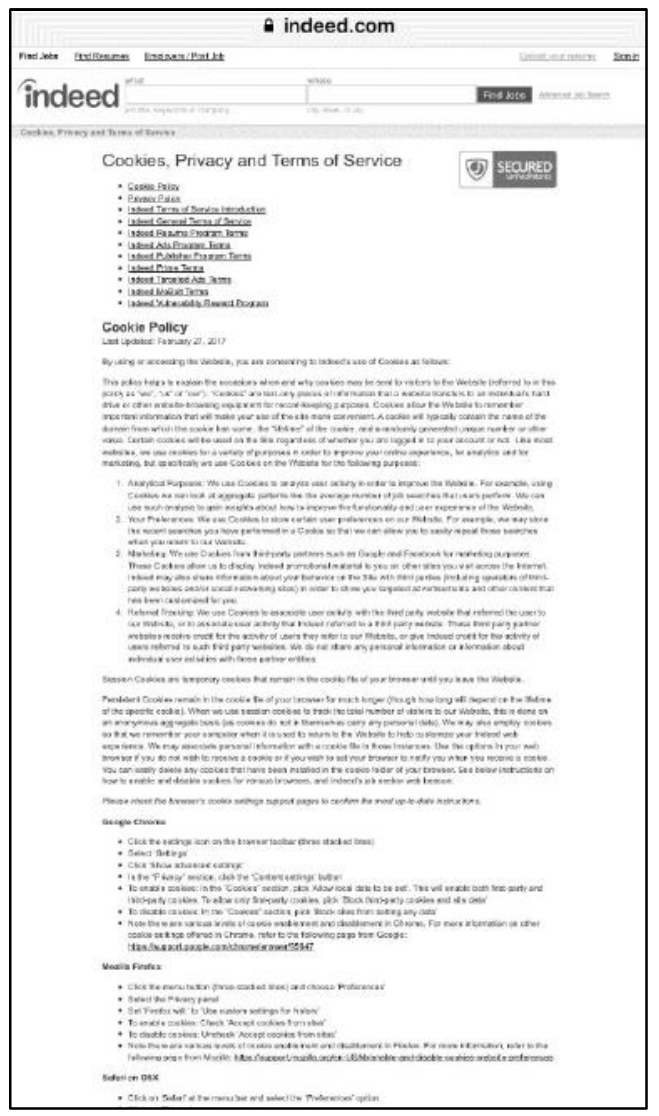

Figure 1. Adverse Readability Issue in Mobile Environment 


\section{Issues in Information Systems}

Volume 18, Issue 4, pp. 179-188, 2017

The second measure is the number of screen swipes. Following the calculations by Leo Qin (Qin, 2016) we estimated the total number of swipes needed to read the text. This was done by adopting the recommended 65 characters per line and 27 lines per screen (the range presented by Qin was 23 lines for a 4.3 inch screen and 30 lines for a 5.5 inch screen, we took the average and rounded up due to a general movement toward larger screens). This gave us a total of 1,755 characters per screen. Using the character count, we were then able to calculate the total number of screens swipes needed and subtracted one since one full screen is presented without needing a swipe, the result was a range of 0 to 90 swipes with an average of 8.5 swipes.

Table 2. Privacy Policy Metrics

\begin{tabular}{|c|c|c|c|}
\hline Metric & Mean & Median & Range \\
\hline \multicolumn{4}{|c|}{ Guideline 1: Use plain, straightforward language. Avoid technical or legal jargon. } \\
\hline 1.1 Flesch Reading Ease & 34.8 & 34.2 & $16.2-57.7$ \\
\hline 1.2 Flesch-Kincaid Reading Level & 14.0 & 14.2 & $9.6-17.3$ \\
\hline \multicolumn{4}{|l|}{ Guideline 2: Use short sentences. Use the active voice. } \\
\hline 2.1 Average Number of Words Per Sentence & 23.7 & 24.0 & $14.4-31.5$ \\
\hline 2.2 Average Number of Sentences Per Paragraph & 2.7 & 2.6 & $1.2-5.5$ \\
\hline 2.3 Fry Test & 15.8 & 16 & $10-17$ \\
\hline 2.4 Percentage of Passive Voice Sentences & $15 \%$ & $14 \%$ & $2 \%-39 \%$ \\
\hline \multicolumn{4}{|c|}{$\begin{array}{l}\text { Guideline 3: Use a format that makes the policy readable, including on smaller screens, such as on a mobile } \\
\text { device. }\end{array}$} \\
\hline 3.1 Predicted Cloze Score & 28 & 28 & $23-36$ \\
\hline 3.2 Number of swipes to read entire text & 8.49 & 7.36 & $0-90.34$ \\
\hline
\end{tabular}

In order to better understand these findings, we used several visualization tools to show results. First we used Readability Studio which allows a group of documents to be analyzed using various readability tests and produces results in a visual format. The results for the Flesch Reading Score and Flesh-Kincaid Reading Ease are similar to those found by Microsoft Word. This helps gain insight by displaying the distribution of the privacy policies for these measures. Figures $\mathbf{2}$ and $\mathbf{3}$ show the results of these tests.

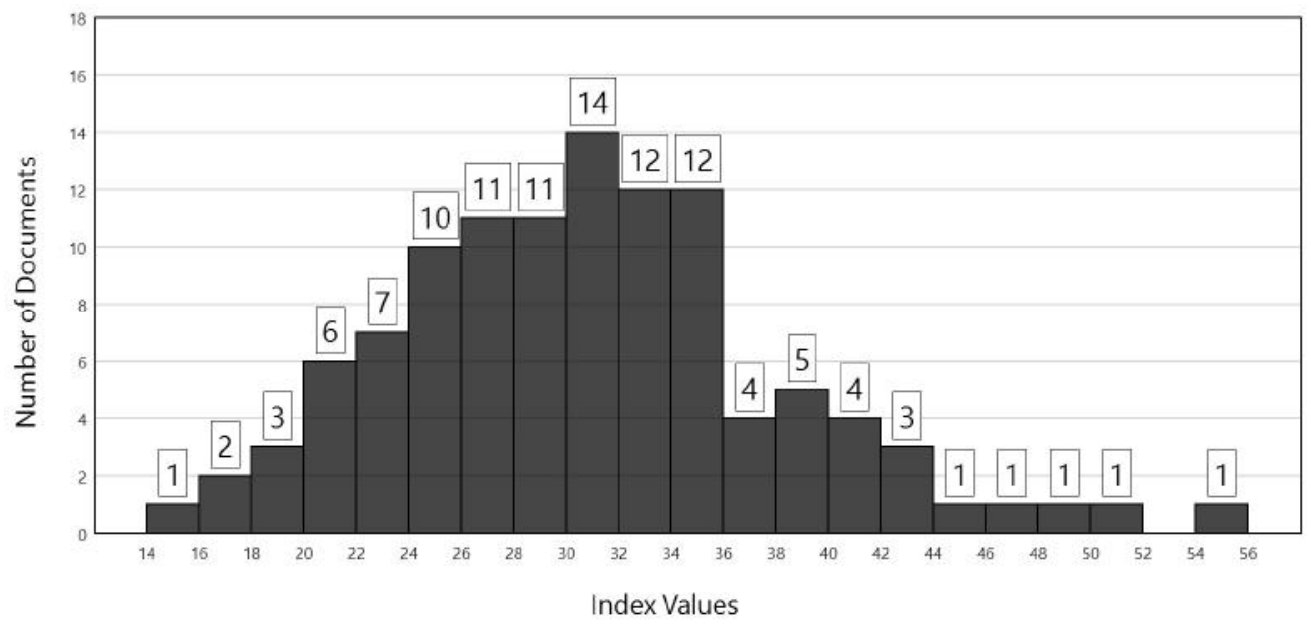

Figure 2. Flesch Reading Ease 


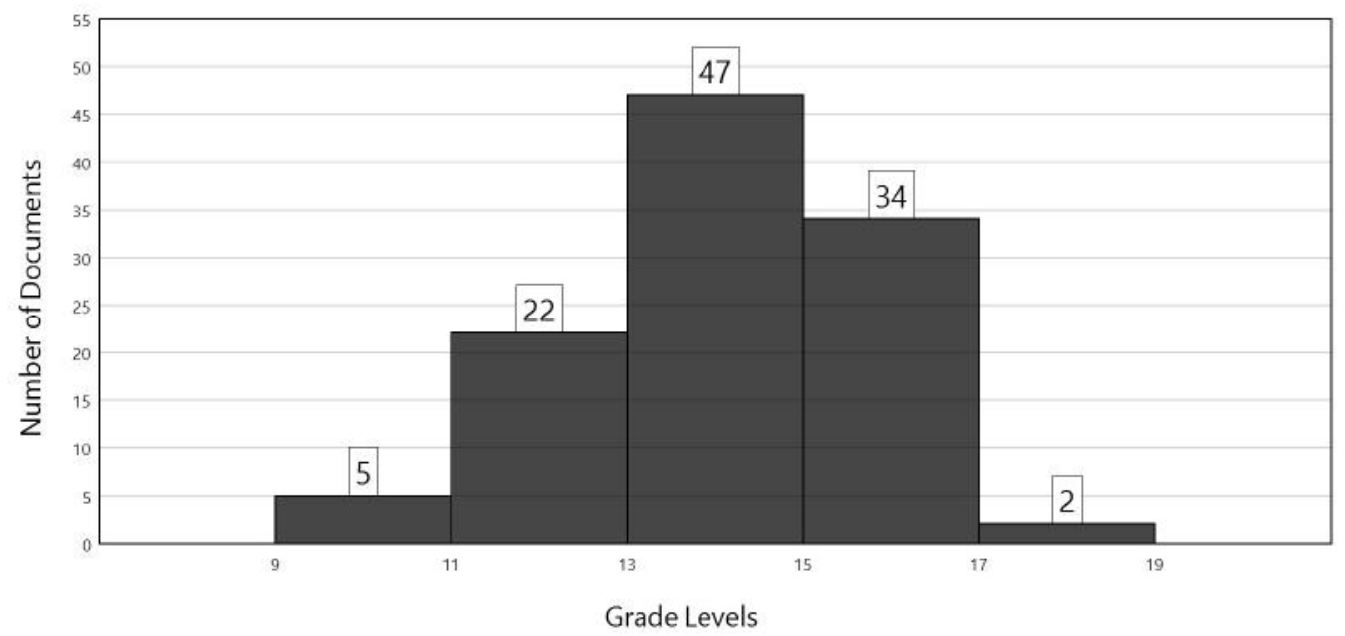

Figure 3. Flesch-Kincaid Reading Level

A Fry Graph is another way to evaluate reading level and visually see the distribution. It measures the text across two basic parameters; syllables and sentences. The assumption is that writing that contains shorter sentences and words with a lower number of syllables is easier to read. Conversely, writing with longer sentences and more syllables per word is more challenging. Figure 4 shows the result of the Fry Test on all of the documents.

The Fry Graph shows that the majority of privacy policies lie in the grade levels 14-17 and above which requires college level reading skills.

Average number of syllables per 100 words

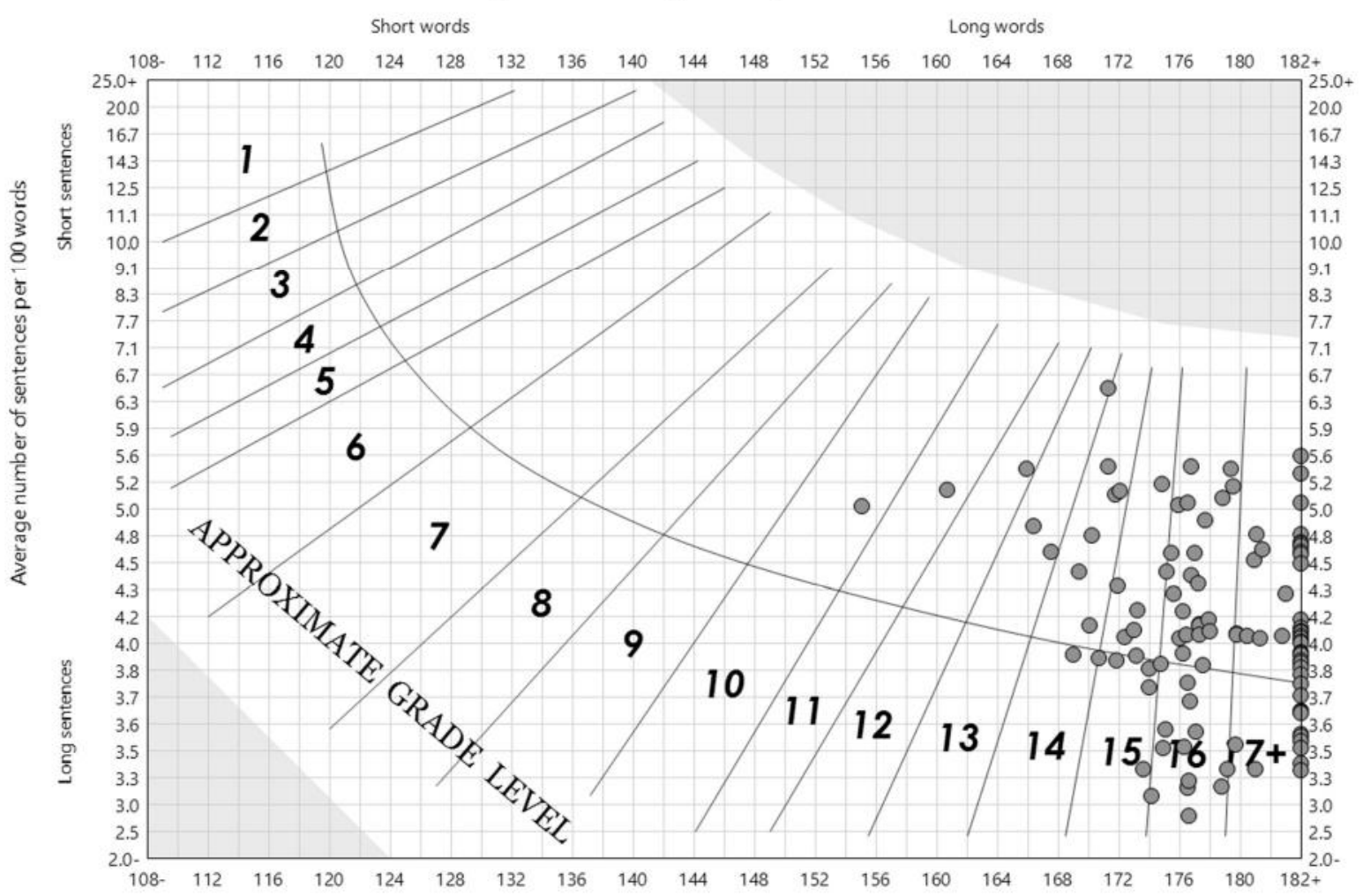

Figure 4. Fry Test 


\section{Issues in Information Systems}

Volume 18, Issue 4, pp. 179-188, 2017

Next, we wanted to see if there was any appreciable difference between privacy policies that recognized the California law in the text of the policy. Out of the 110 documents, 41 specifically mention or include sections for California residents. We did not actually check the documents to see if they met the criteria required by California law, for example if they had a clause explaining how consumers are notified of changes in the privacy policy. We simply searched the documents for "California" and made sure it was not referenced as an address.

The documents were divided into two groups - California and Non-California and the results are shown in Table 3 and Table 4. For the California documents, the measures for Guideline 1 are actually higher, indicating that they use less straightforward English. In the measures for Guideline 2, the average words per sentence and sentences per paragraph were higher for the California set, and the Fry test remained the same, while the passive voice was used less frequently. The measures for Guideline 3 had a predicted average Cloze score the same for both groups, while the number of swipes needed averaged 11.35 for the California documents yet only 6.78 for the non-California documents.

Table 3. California Documents Results $(\mathrm{n}=41)$

\begin{tabular}{|l|c|c|c|}
\hline Metric & Mean & Median & Range \\
\hline Guideline 1: Use plain, straightforward language. Avoid technical or legal jargon. \\
\hline 1.1 Flesch Reading Ease & 34.4 & 33.6 & $19.0-53.0$ \\
\hline 1.2 Flesch-Kincaid Reading Level & 14.2 & 14.2 & $10.5-17.0$ \\
\hline Guideline 2: Use short sentences. Use the active voice. \\
\hline 2.1 Average Number of Words Per Sentence & 24.1 & 24.3 & $17.3-31.5$ \\
\hline 2.2 Average Number of Sentences Per Paragraph & 2.8 & 2.7 & $1.8-5.5$ \\
\hline 2.3 Fry Test & 16 & 16 & $11-17$ \\
\hline 2.4 Percentage of Passive Voice Sentences & $14 \%$ & $13 \%$ & $4 \%-24 \%$ \\
\hline $\begin{array}{l}\text { Guideline 3: Use a format that makes the policy readable, including on smaller screens, such as on a mobile } \\
\text { device. }\end{array}$ & 28 & 27 & $23-33$ \\
\hline 3.1 Predicted Cloze Score & 11.35 & 8.80 & $1.36-90.34$ \\
\hline 3.2 Number of swipes to read entire text
\end{tabular}

Table 4. Non-California Documents Results ( $\mathrm{n}=69$ )

\begin{tabular}{|c|c|c|c|}
\hline Metric & Mean & Median & Range \\
\hline \multicolumn{4}{|c|}{ Guideline 1: Use plain, straightforward language. Avoid technical or legal jargon. } \\
\hline 1.1 Flesch Reading Ease & 35.1 & 34.4 & $16.2-57.7$ \\
\hline 1.2 Flesch-Kincaid Reading Level & 13.9 & 14.3 & $9.6-17.3$ \\
\hline \multicolumn{4}{|l|}{ Guideline 2: Use short sentences. Use the active voice. } \\
\hline 2.1 Average Number of Words Per Sentence & 23.5 & 23.8 & $14.4-31.0$ \\
\hline 2.2 Average Number of Sentences Per Paragraph & 2.6 & 2.5 & $1.2-4.9$ \\
\hline 2.3 Fry Test & 16 & 16 & $10-17$ \\
\hline 2.4 Percentage of Passive Voice Sentences & $16 \%$ & $14 \%$ & $2 \%-39 \%$ \\
\hline \multicolumn{4}{|c|}{$\begin{array}{l}\text { Guideline 3: Use a format that makes the policy readable, including on smaller screens, such as on a mobile } \\
\text { device. }\end{array}$} \\
\hline 3.1 Predicted Cloze Score & 28 & 28 & $23-36$ \\
\hline 3.2 Number of swipes to read entire text & 6.78 & 6.12 & $0-66.69$ \\
\hline
\end{tabular}




\section{Issues in Information Systems}

Volume 18, Issue 4, pp. 179-188, 2017

Using the same visual results, we provided earlier, Figure 5 shows the results for the Flesch Reading Ease formula for the documents that specifically mention California versus all of the others. There doesn't appear to be a significant difference between the two group's distributions.

Similarly, in Figure 6, we charted the two groups using the Fry Graph, and again noticed very little difference in how the documents were distributed with respect to words and sentence characteristics.

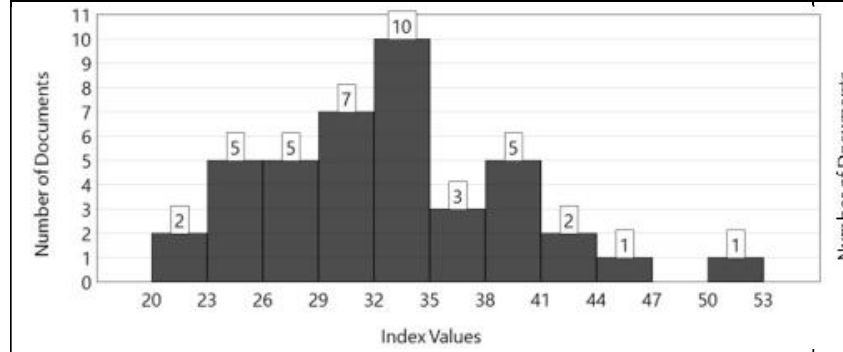

California Documents

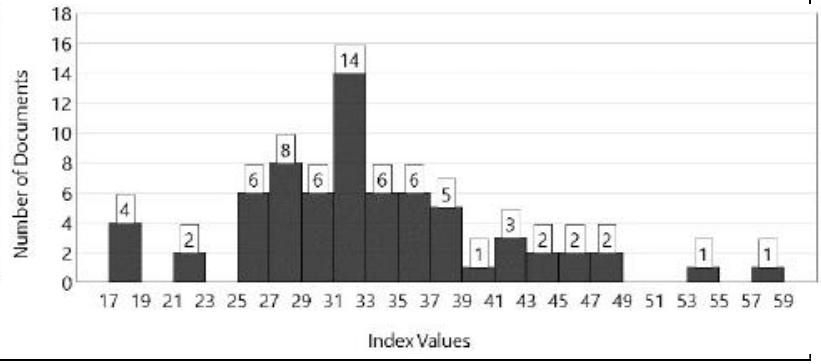

Non-California Documents

Figure 5. Flesh Reading Ease

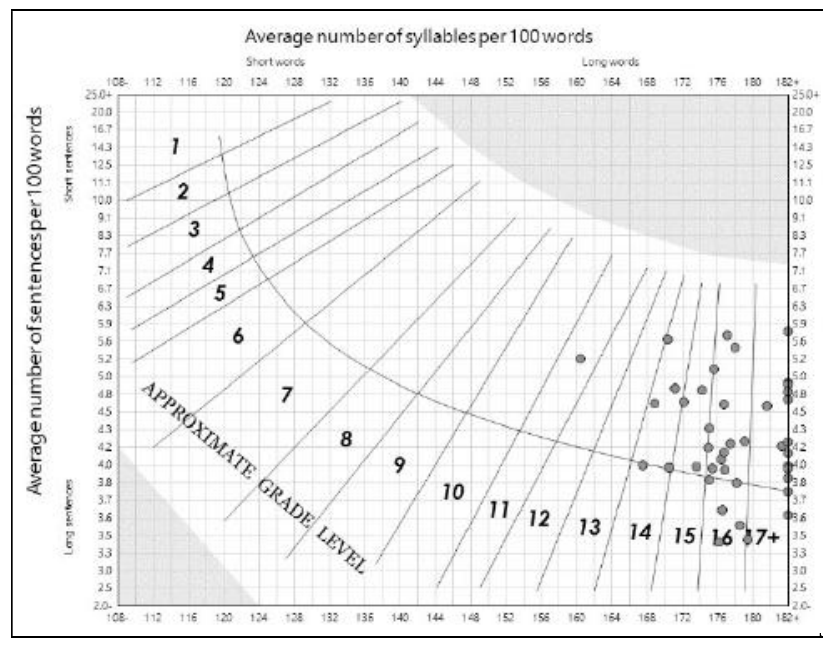

California Documents

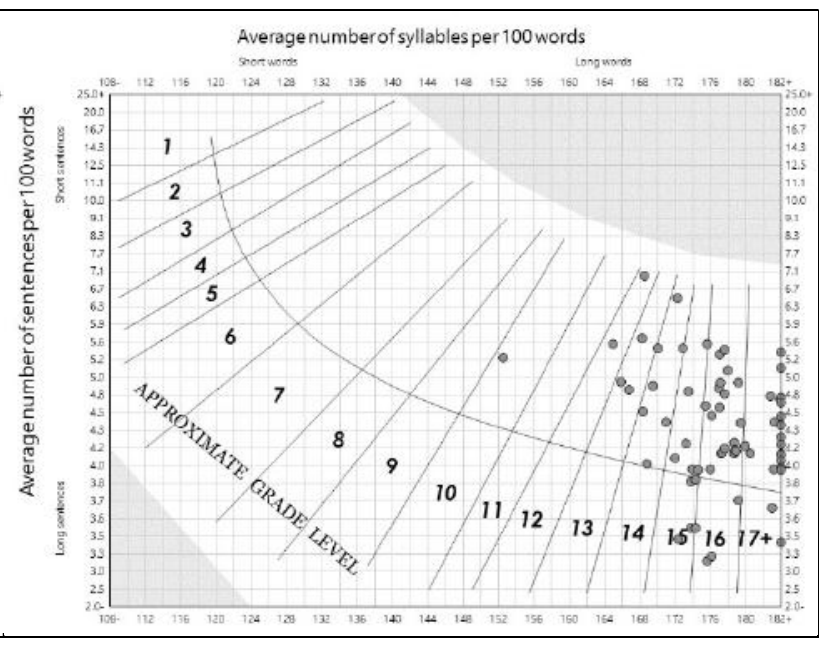

Non-California Documents

Figure 6. Fry Graphs

Overall we see that California-specific privacy statements were, on average, slightly worse in relation to the overall guidelines.

\section{SUMMARY}

Based upon the results of our study, a majority of the privacy policies for the top business applications fail to meet the guidelines as set forth by the California Attorney General's Office. They are far from simplistic, with most requiring a minimum of a high-school diploma and some requiring at least a university degree. They are lengthy, with an average of over 3,000 words, and average sentence length of 24 words. Finally, many are unreadable on the very device on which the application is designed to operate. The test requires magnification and a significant number of swipes both left and right, as well as up and down, making them extremely cumbersome to read.

Some suggest that the privacy policy is not written for the end user, but to address the legal obligations of an organization to disclose its privacy practices as they relate to the interactions with users (Singh, 2011). Organizations need to balance the requirements of writing plain English documents that are consumable by the customers using those 


\section{Issues in Information Systems}

Volume 18, Issue 4, pp. 179-188, 2017

apps with the necessity that the document represents a legal document that will hold up in court. This research suggests that the emphasis has been more on the latter and more work needs to be done to assist the consumer.

Next, we compared those policies that specifically mentioned California law, with the belief that those privacy statements may have already been adjusted to meet the guidelines as set forth by the California Attorney General's Office. However, what we found was that by all measures the California group was either worse off or the same in their results. This suggests that while companies may be putting in language to satisfy California law, they appear to not be following the Attorney General's guidelines to make those policies easier to read. This could be due to either a) the difficulty in achieving the guidelines while at the same time trying to add wording to satisfy California law or b) the Attorney General's guidelines were simply not taken into account when the privacy policy was developed.

This work has several limitations that we intend to address in future work. First, it evaluates only applications aimed at business users. One could certainly argue that it is expected that a business user will have a higher level of education than the average user, and as such, these policies can be more complex. Second, while we have evaluated these policies from a user perspective, we have concluded that companies need to balance both user and legal requirements. It could be that the legal requirements will not be met with simpler privacy policies, and we have not evaluated these policies on legal grounds.

We plan to continue this work by using text analytics and qualitative data analysis to study the contents of the agreements in depth. We want to try to identify the commonality between these documents to create recommendations about the structure and content of these documents that tries to balance the legal requirements that an organization must meet with the needs of the user to understand what the privacy policy will do for them. We intend to further expand the categories of documents analyzed to see if other categories produce different results.

\section{REFERENCES}

App Store Review Guidelines. (2017, May 5). Retrieved from Apple Inc.: https://developer.apple.com/appstore/review/guidelines/

California State Legislature. (2003). Internet Privacy Requirements. Retrieved from California Legislative Information:

https://leginfo.legislature.ca.gov/faces/codes_displaySection.xhtml?lawCode=BPC\&sectionNum=22575

Cutts, M. \&. (2004). Oxford guide to plain English. New York: Oxford University Press.

Felker, D. B. (1981). Guidelines for Document Designers. Retrieved from http://files.eric.ed.gov/fulltext/ED221866.pdf

Hall, W. E. (1986). The Cloze Procedure and Software Comprehensibility Measurement. IEEE Transactions on Software Engineering, 12(5), 608.

Kamal D. Harris, Attorney General, California Department of Justice. (2014, May). Making your Privacy Practices Public. Retrieved from https://oag.ca.gov/sites/all/files/agweb/pdfs/cybersecurity/making_your_privacy_practices_public.pdf

Mobile app Industry Agrees to Privacy Standards in California. (2012). Computer and Internet Lawyer, 29(5), 20 21.

Oleander Software, L. (2017). Readability Studio. Retrieved from http://www.oleandersolutions.com/readabilitystudio.html

Plain English. (2017, May 12). Retrieved from Wikipedia: https://en.wikipedia.org/wiki/Plain_English 


\section{Issues in Information Systems}

Volume 18, Issue 4, pp. 179-188, 2017

Qin, L. (2016, July 1). How Many Miles Will You Scroll? Retrieved from https://www.leozqin.me/how-many-mileswill-you-scroll/

Rainie, L. (2017, July 1). The State of Privacy in Post-Snowden America. Retrieved from Pew Research Center: http://www.pewresearch.org/fact-tank/2016/09/21/the-state-of-privacy-in-america/

Singh, R. I. (2011). A user-centric evaluation of the readability of privacy policies in popular web sites. Information Systems Frontiers, 13(4), 501-514.

Taylor, W. L. (1953). Cloze Procedure: A New Tool for Measuring Readability. Journalism Quarterly, 30, 415-430.

Vincent, S. (2014, August 4). Sentence Length: Why 25 Words is Our Limit. Retrieved May 2017, from Inside UK.GOV: https://insidegovuk.blog.gov.uk/2014/08/04/sentence-length-why-25-words-is-our-limit/ 